\title{
Adaptive optics with an infrared pyramid wavefront sensor
}

Charlotte Z. Bond, Peter Wizinowich, Mark Chun, Dimitri Mawet, Scott Lilley, et al.

Charlotte Z. Bond, Peter Wizinowich, Mark Chun, Dimitri Mawet, Scott Lilley, Sylvain Cetre, Nemanja Jovanovic, Jacques-Robert Delorme, Edward Wetherell, Shane Jacobson, Charles Lockhart, Eric Warmbier, James K. Wallace, Donald N. Hall, Sean Goebel, Olivier Guyon, Cedric Plantet, Guido Agapito, Christophe Giordano, Simone Esposito, Bruno Femenia-Castella, "Adaptive optics with an infrared pyramid wavefront sensor," Proc. SPIE 10703, Adaptive Optics Systems VI, $107031 Z$ (10 July 2018); doi: 10.1117/12.2314121

EDent: SPIE Astronomical Telescopes + Instrumentation, 2018, Austin, Texas, United States 


\title{
Adaptive optics with an infrared Pyramid wavefront sensor
}

\author{
Charlotte Z. Bond ${ }^{\mathrm{a}}$, Peter Wizinowich ${ }^{\mathrm{b}}$, Mark Chun ${ }^{\mathrm{a}}$, Dimitri Mawet ${ }^{\mathrm{c}}$, Scott Lilley ${ }^{\mathrm{b}}$, Sylvain \\ Cetre $^{\mathrm{b}}$, Nemanja Jovanovic ${ }^{\mathrm{c}}$, Jacques-Robert Delorme ${ }^{\mathrm{c}}$, Edward Wetherell ${ }^{\mathrm{b}}$, Shane Jacobson ${ }^{\mathrm{a}}$, \\ Charles Lockhart ${ }^{\mathrm{a}}$, Eric Warmbier ${ }^{\mathrm{a}}$, James K. Wallace ${ }^{\mathrm{f}}$, Donald N. Halla ${ }^{\mathrm{a}}$, Sean Goebel ${ }^{\mathrm{a}}$, \\ Olivier Guyon ${ }^{\mathrm{d}}$, Cedric Plantet ${ }^{\mathrm{e}}$, Guido Agapito ${ }^{\mathrm{e}}$, Christophe Giordano ${ }^{\mathrm{e}}$, Simone Esposito ${ }^{\mathrm{e}}$, \\ and Bruno Femenia-Castella ${ }^{b}$ \\ ${ }^{a}$ Institute for Astronomy, University of Hawaii, 640 N. Aohoku Place, Hilo, HI 96720, USA. \\ ${ }^{b}$ W. M. Keck Observatory, 65 - 1120 Mamalahoa Hwy., Kamuela, HI 96743, USA. \\ cDepartment of Astronomy, California Institute of Technology, Pasadena, CA 91106, USA \\ ${ }^{\mathrm{d} S}$ Subaru Telescope NAOJ, 650 N. Aohoku Place, Hilo, HI 96720, USA \\ e INAF Osservatorio Astronomico di Arcetri, 50125 Firenze, Italy \\ ${ }^{\mathrm{f} J e t}$ Propulsion Laboratory, California Institute of Technology, Pasadena, CA 91109, USA
}

\begin{abstract}
Wavefront sensing in the infrared is highly desirable for the study of M-type stars and cool red objects, as they are sufficiently bright in the infrared to be used as the adaptive optics guide star. This aids in high contrast imaging, particularly for low mass stars where the star-to-planet brightness ratio is reduced. Here we discuss the combination of infrared detector technology with the highly sensitive Pyramid wavefront sensor (WFS) for a new generation of systems. Such sensors can extend the capabilities of current telescopes and meet the requirements for future instruments, such as those proposed for the giant segmented mirror telescopes.

Here we introduce the infrared Pyramid WFS and discuss the advantages and challenges of this sensor. We present a new infrared Pyramid WFS for Keck, a key sub-system of the Keck Planet Imager and Characterizer (KPIC). The design, integration and testing is reported on, with a focus on the characterization of the SAPHIRA detector used to provide the $\mathrm{H}$-band wavefront sensing. Initial results demonstrate a required effective read noise $<1 \mathrm{e}^{-}$at high gain.
\end{abstract}

Keywords: Wavefront sensing, adaptive optics, Pyramid wavefront sensor, infrared

\section{INTRODUCTION}

Wavefront sensing in the infrared has several advantages over visible wavefront sensing for particular science cases. Systems of particular scientific interest, such as M dwarf stars, are bright enough in the infrared to use as $\mathrm{AO}$ guide stars, providing high quality $\mathrm{AO}$ correction around the science target. ${ }^{1}$ Previously such stars have been difficult to observe at the contrasts and resolution required for exoplanet detection due to their faintness at optical wavelengths.

Developments in wavefront sensing motivated by the future demands of astronomy have led to the emergence of the Pyramid wavefront sensor (WFS) ${ }^{2}$ as a prime candidate for new AO systems. This sensor has shown improved performance over industry standard sensors, such as the Shack Hartmann WFS, exhibiting greater sensitivity within the correction band and reduced susceptibility to aliasing. ${ }^{3}$ Demonstration on the Large Binocular Telescope (LBT), ${ }^{4,5}$ Subaru's extreme AO system SCExAO $^{6}$ and the Magellan telescope ${ }^{7}$ have provided compelling results with visible sensors. Combining infrared technology with the highly sensitive Pyramid WFS will provide new AO systems ideally suited for the study of faint red objects.

In this paper we present an overview of the infrared Pyramid WFS, examining its behavior, advantages and challenges within the framework of implementing a new infrared wavefront sensor on the Keck telescope.

(Send correspondence to C. Z. Bond)

E-mail: czbond@hawaii.edu

Adaptive Optics Systems VI, edited by Laird M. Close, Laura Schreiber,

Dirk Schmidt, Proc. of SPIE Vol. 10703, 107031Z • ( 2018 SPIE

CCC code: $0277-786 \mathrm{X} / 18 / \$ 18 \cdot$ doi: $10.1117 / 12.2314121$

Proc. of SPIE Vol. 10703 107031Z-1 
This forms part of the Keck Planet Imager and Characterizer (KPIC) project, ${ }^{8}$ in which a near infrared, high order Pyramid wavefront sensor (WFS) will be installed on Keck II, with the aim of providing high resolution AO correction for the study of exoplanets around M-type stars and planet formation in obscured star forming regions. This system will extend the capabilities of the Keck telescope and provide insight into such instruments for future AO systems, such as those planned for the giant segmented mirror telescopes.

We report on the integration and testing of the near infrared Pyramid WFS designed for KPIC, including integration in the laboratory and first system tests. A crucial step towards the realization of infrared wavefront sensors has been the recent developments in low noise infrared detectors. Previous infrared systems have been limited to bright targets due to the high read noise of the detectors, such as the first near-infrared Pyramid WFS demonstrated on sky with the Calar Alto AO system. ${ }^{9}$ In the case of KPIC the wavefront sensing is provided in H-band using the low noise e-APD array SAPHIRA, ${ }^{13}$ an infrared detector extensively developed by Leonardo and the University of Hawaii. We report here on the testing and characterization of this state of the art camera within the KPIC and Keck AO systems.

\section{INFRARED PYRAMID WAVEFRONT SENSING}

\subsection{The Pyramid WFS}

In recent years the Pyramid WFS has emerged as a prime candidate for high resolution, high contrast AO systems. The classical sensor comprises a 4 sided prism, re-imaging optics and a detector. Light from the telescope is focused onto the tip of the prism, which projects four images of the telescope pupil onto the detector via the re-imaging optics. ${ }^{2}$ Any distortion of the incoming wavefront changes the distribution of light within the
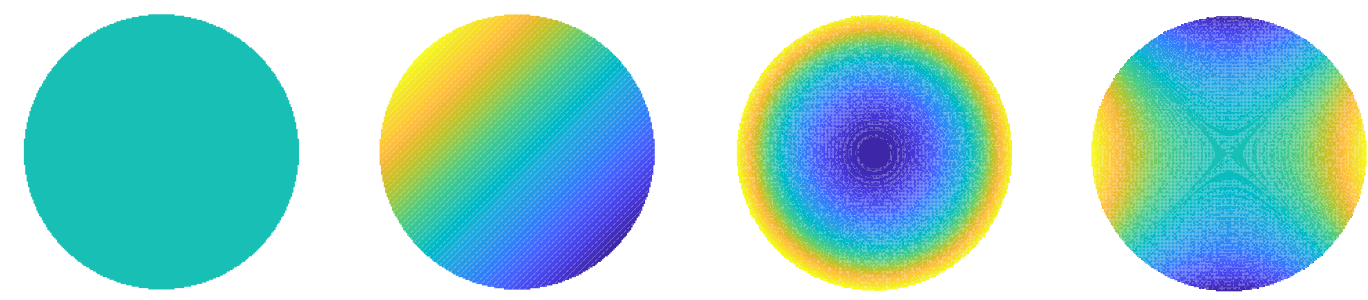

(a) Low order wavefront aberrations.
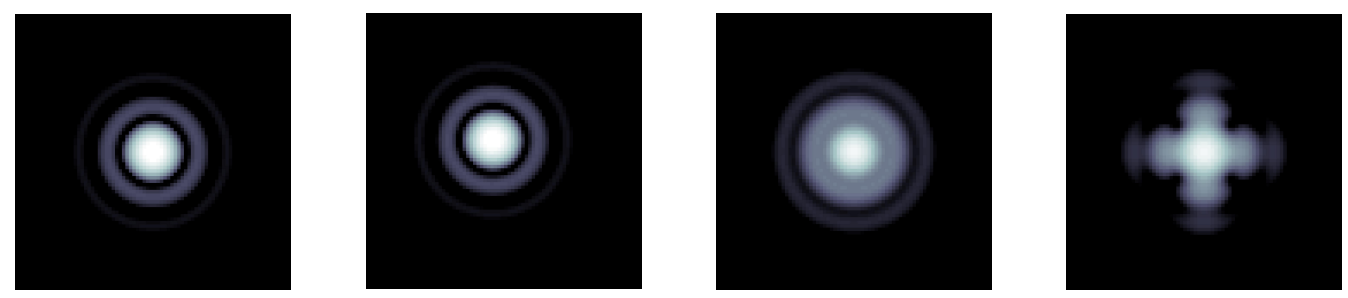

(b) Focal plane images for low order wavefront aberrations.
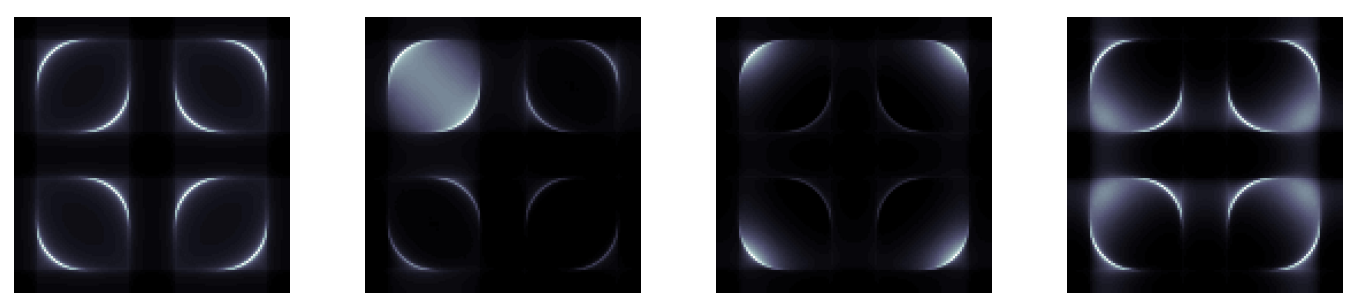

(c) Pyramid WFS signals for low order wavefront aberrations.

Figure 1: Effect of low order phase aberrations on the wavefront (a), focal plane (b) and Pyramid WFS signal (c). From left to right: flat, tip-tilt, focus, and astigmatism. 
four pupil images. Figure 1 illustrates this, showing the wavefront, focal plane image and corresponding Pyramid WFS signals for some low order aberrations.

The Pyramid WFS demonstrates an increased sensitivity to wavefront aberrations compared to the commonly used Shack-Hartmann WFS. ${ }^{3}$ However, the sensitivity comes at the expense of a small range: the sensor quickly becomes non-linear and can saturate with small wavefront aberrations. Consequently the Pyramid WFS is used within a closed AO loop and often modulation is necessary to increase the range for optimal correction. Under these conditions visible Pyramid WFSs have been used to provide the wavefront sensing for AO systems on the Large Binocular Telescope, ${ }^{4,5}$ the Subaru extreme AO system SCExAO, ${ }^{6}$ and Magellan AO system. ${ }^{7}$ These systems have demonstrated the performance of the Pyramid WFS, producing a compelling case for the use of this sensor for future systems, such as the first light instruments of the next generation of giant segmented mirror telescopes, and upgrades to existing instruments.

\subsection{Wavefront sensing in the infrared}

Infrared wavefront sensing is particularly appealing for science cases which are bright in the infrared but faint in the visible. Traditional wavefront sensing, such as the current Keck AO system, is done in the visible. For science cases involving faint and red natural guide stars, this requires off-axis guide stars or LGS for visible wavefront sensing, as the target stars are not bright enough in the visible. However, infrared wavefront sensing would allow the use of the science object as the guide star, providing optimal $\mathrm{AO}$ correction and high resolution imaging of the target. Such motivation is described in greater detail in Mawet et al. (2016). ${ }^{8}$

Although the choice of the wavefront sensing wavelength is primarily dictated by the science case, there are additional benefits for Pyramid wavefront sensing when compared with the visible. These advantages stem from the small range and non-linear behavior which can impact the performance of the Pyramid WFS, especially at shorter wavelengths. Here we discuss two effects:

1. A reduction in sensitivity. The highly sensitive state of the Pyramid WFS equates to the case of a diffraction limited PSF on the tip of the Pyramid, and is only maintained within small deviations from this state. Distortions of the PSF on the tip of the Pyramid reduce the sensitivity.

2. Small measurement range. The non-modulated Pyramid WFS has a limited range and quickly saturates. Wavefront errors out of this range will be underestimated by the sensor.

Both of these effects have been seen to impact the performance in the visible, requiring modulation to mitigate particularly the limited range of the sensor. However, both effects can also be reduced by going to longer wavelengths: the PSF is better corrected and the range is larger.

To illustrate the impact of these effects on the Pyramid performance in closed loop end-to-end simulations using a V-band and H-band Pyramid wavefront sensor were carried out, for a range of seeing conditions and modulation amplitudes. These results are summarized in figure 2. In the case of the visible WFS the nonmodulated case does not provide sufficient correction due to the effects described above. Modulation is required to extend the range of the sensor, particularly for small $r_{0}$ where the incoming wavefront errors are larger. In comparison the H-band WFS sees little difference between the different modulation amplitudes, with small improvements with increased modulation at small $r_{0}$; and with no modulation in good seeing conditions. Using a Pyramid WFS in the infrared can require less modulation and allow for use in it's most sensitive state (without modulation).

Another aspect which can impact the PSF sent to the Pyramid are any non-common path aberrations (NCPAs). These are traditionally dealt with by offsetting the wavefront sensor to produce the best possible image on the science camera, with a distorted PSF on the WFS. The impact of this can be quite significant in the visible, as even small NCPAs can saturate the sensor. If the NCPA is beyond the range of the sensor the loop is no longer stable. ${ }^{10}$ This is avoided by using a larger modulation. Sensing in the infrared provides a larger range, resulting in either:

- less strict requirements on NCPA in the design phase.

- or, a smaller minimum modulation for a given setup (and fixed NCPA). 


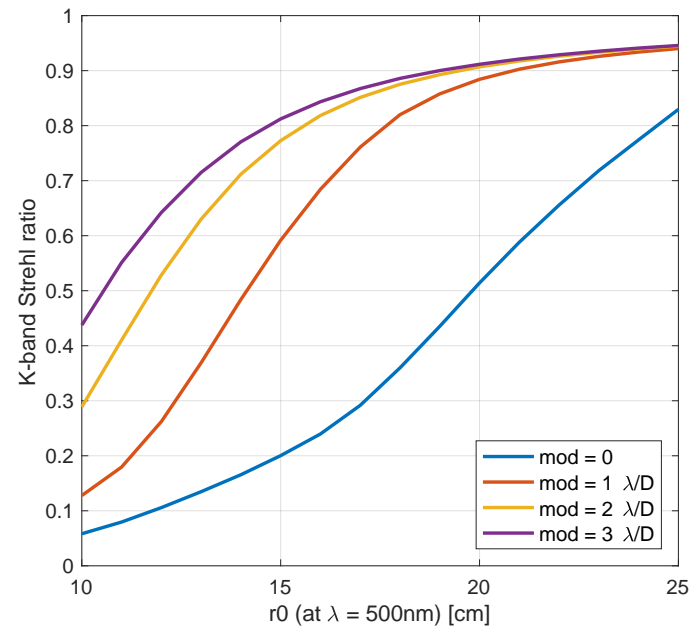

(a) Wavefront sensing in V-band

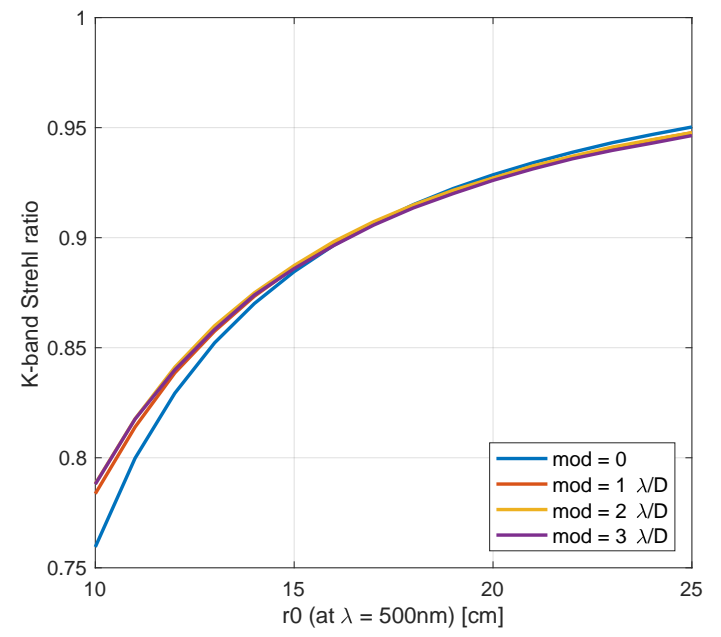

(b) Wavefront sensing in H-band

Figure 2: Simulated performance (K-band Strehl ratio) of a $20 x 20$ AO system with $D=10 \mathrm{~m}$. The wavefront sensing is done with a Pyramid wavefront sensor in V-band (a) and H-band (b).

\subsection{The Keck Planet Imager and Characterizer and a near-infrared Pyramid WFS for Keck}

The Keck Planet Imager and Characterizer (KPIC) is a new instrument for the Keck obseveratory which aims to provide high resolution images for the study of cool, red objects, in particular:

- M-dwarf systems and associated exo-planets.

- Young planets in proto-planetary discs.

Within this system a near-infrared Pyramid wavefront sensor will be implemented on the Keck II AO bench to provide the high resolution $\mathrm{AO}$ correction required. Carrying out the wavefront sensing in the infrared will allow for the use of the target as the WFS natural guide star, optimizing the correction for these particular science cases. For such cases the use of the infrared will increase the sky coverage of the AO system. ${ }^{1,8}$

Within the KPIC system the infrared Pyramid WFS (PWS) will be used with NIRC2, a near-infrared imager utilizing a vortex coronograph; and NIRSPEC, a near infrared spectrograph. In conjunction with the Pyramid WFS KPIC includes a new Fiber Injection Unit (FIU) which will be used to feed NIRSPEC to provide high resolution spectroscopy. ${ }^{11}$

The Pyramid WFS for KPIC will be in addition to the current Keck WFS, a $20 \times 20$ visible Shack-Hartmann. Initially the Pyramid WFS will drive the current Keck deformable mirror (DM), but in a second phase a higher order deformable mirror will be added to KPIC to provide higher order correction for the FIU and Pyramid WFS.

\section{DESIGN CONSIDERATIONS}

Here we discuss the design of a Pyramid WFS, using the particular example of the infrared Pyramid WFS for KPIC. In this case the key requirements are:

- Wavefront sensing carried out in H-band.

- A maximum frame rate of $1.5 \mathrm{kHz}$. 
- An infrared detector, with readout noise $<1 \mathrm{e}^{-}$.

- A range of modulation up to an amplitude of $6 \frac{\lambda}{D}$, synchronized with the WFS camera.

\subsection{Wavefront sampling}

A key design consideration for such a system is the sampling of the wavefront. The lower limit on the sampling is set by the number of DM actuators across the pupil. In the case of KPIC we require at least 32 pixels across the pupil to match the 32 actuators of the DM included in the second phase. However, for the Pyramid WFS it is often advantageous to over sample the pupil, to avoid some insensitivity to higher spatial frequencies. This insensitivity manifests itself due to the positioning of the four pupils on the detector: if the pupils aren't separated by an integer number of pixels the sampling points lie at different locations across the four different pupils. Achieving a seperation of an integer number of pixels requires very tight tolerances on the angles of the Pyramid faces. Another solution is to slightly over-sample the pupils. For the Keck Pyramid a sampling of 40 pixels was chosen for this reason. Figure 3 shows simulations of the Keck Pyramid as the pupil positions are shifted from an integer seperation. As the shift increases the insensitivity to high spatial frequencies increases, requiring removal of

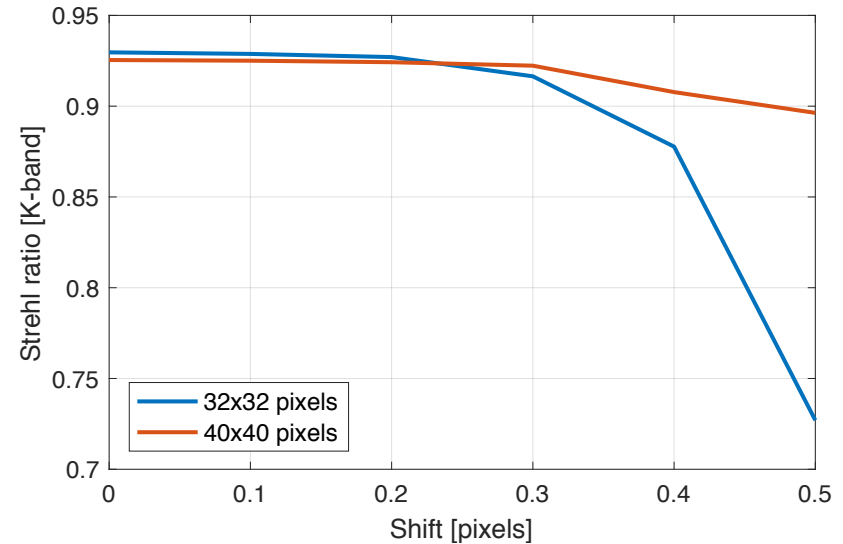

Figure 3: Simulated performance (Strehl ratio in Kband) of the Keck Pyramid WFS vs. shift in the pupil seperation from an integer number of pixels. The results are shown for a sampling of 32 pixels, matching the number of DM actuators, and an oversampled case of 40 pixels. these modes from the reconstructor for a stable closed loop and leading to a drop in performance. The impact of this effect is smaller in the the over-sampled case, even for the worst case of a $\frac{1}{2}$ pixel shift, and so the Keck Pyramid was designed with 40 pixels across the pupil.

The choice of sampling, along with the size of the detector pixels, will set the size of the pupil images and hence set requirements for the f-ratio of the incoming beam and focal length of the re-imaging lens for the WFS.

\subsection{Optical and mechanical design}

Here we briefly outline the design of the Keck Pyramid WFS. For a more detailed description please see Lilley et al. (2018). ${ }^{12}$ The optical layout of the WFS is illustrated in figure 4. The key elements of the optical design are:

- An input beam provided by Keck AO with f/13.66.

- Two OAP (off-axis parabola) relays which provide two pupil planes, one for the second phase DM (currently a flat mirror) and one for the WFS modulator.

- Collimating and focusing lenses provide an f/56.5 beam at the Pyramid WFS.

- A translation stage attached to the collimating lens allows for adjustment of the focus onto the Pyramid WFS, providing maximum conjugation between the WFS and different science instruments.

- The Pyramid WFS, consisting of the two roof prisms, re-imaging lens to provide the correct sampling and SAPHIRA camera.

The system is designed to produce four pupil images on the detector, each 40 pixels across and separated by 64.5 pixels $(1$ pixel $=24 \mathrm{um})$. The four pupils fit within a $128 \times 128$ sub-array of the SAPHIRA, allowing for operation of the detector at high frame rates. The wavefront sensing will be carried out in H-band, requiring 


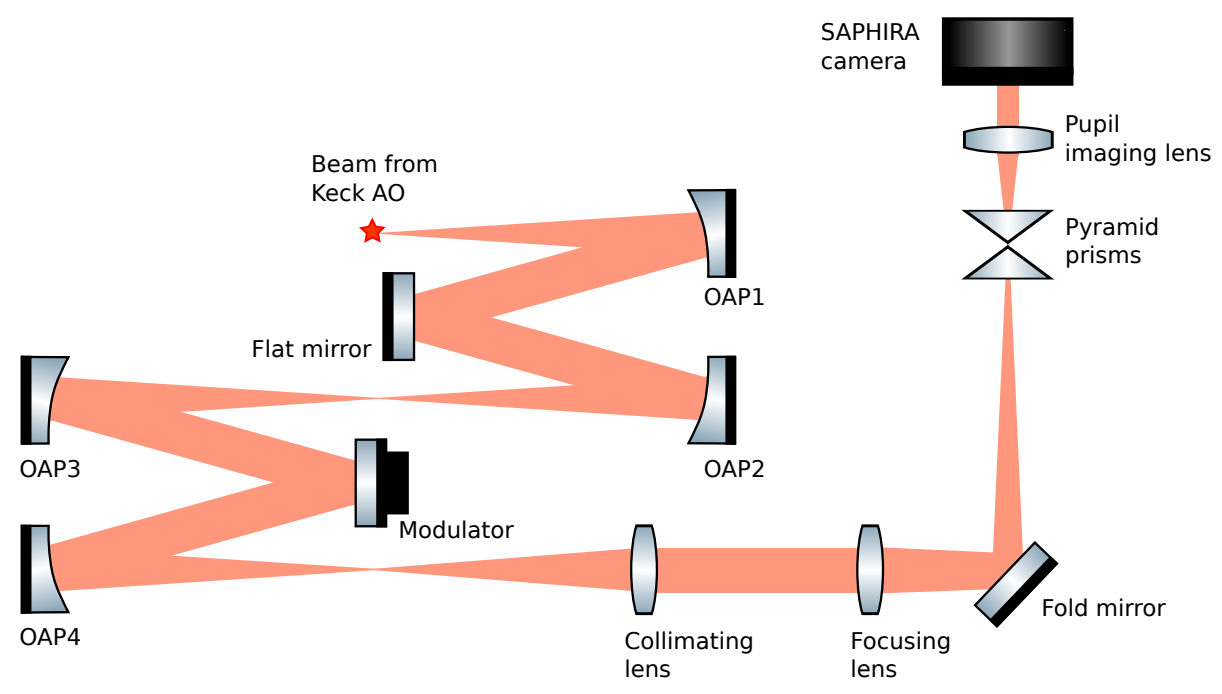

Figure 4: Diagram of the optical layout for the KPIC infrared Pyramid wavefront sensor. The optical setup consists of two OAP (off-axis parabola) relays, each providing a pupil plane; a collimating lens; a focusing lens; the two roof prisms of the Pyramid WFS; a re-imaging lens and the WFS detector.

an H-band filter to avoid large chromatic aberrations, as both $\mathrm{H}$ - and J-band are reflected at the FIU/PWS dichroic.

The mechanical design of KPIC is strongly influenced by the small amount of space available on the Keck AO bench. Consequently the optics for both the PWS and FIU are installed on individual vertical plates, which are themselves attached to a common baseplate. This allows for installation of the plates individually.

An overview of the mechanical design is shown in figure 5, the full instrument (a) and PWS plate (b). The light from Keck AO is delivered to the PWS plate from the FIU plate, via the first OAP and first flat mirror

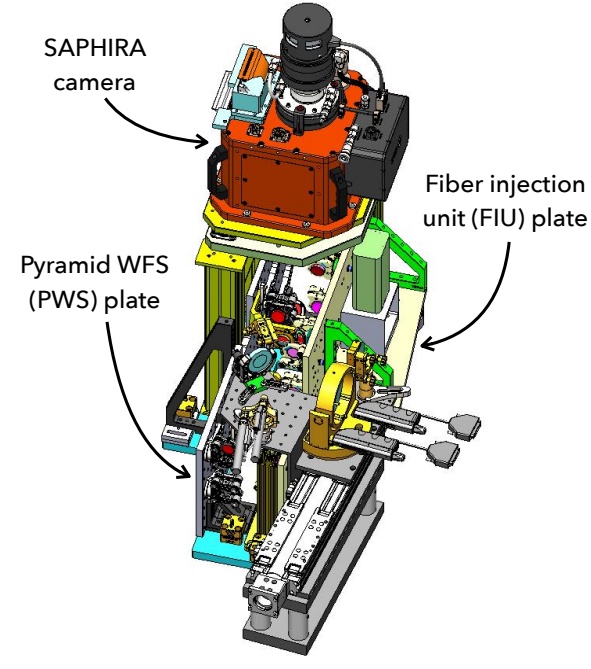

(a) KPIC

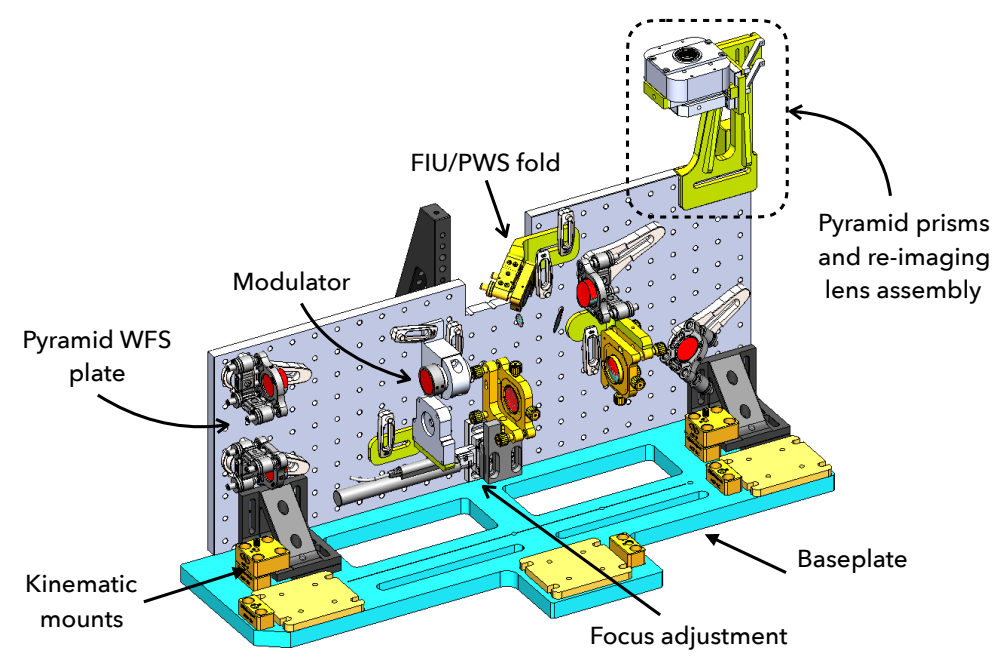

(b) Keck Pyramid WFS

Figure 5: Models showing the opto-mechanical design of KPIC. An overview of the instrument is shown (a) as well as the details of the PWS plate (b). 
(deformable mirror in phase 2) of the optical design shown in figure 4. A dichroic delivers $\mathrm{J}$ and $\mathrm{H}$ band light to the PWS plate. Further details of the mechanical and optical design can be found in Lilley et al. (2018). ${ }^{12}$

\subsection{Detector}

The practical implementation of infrared wavefront sensors has only recently become feasible due to advancements in infrared detectors. For the Keck Pyramid WFS camera the SAPHIRA detector ${ }^{13}$ is used in order to meet the key requirements: a read noise of $<1 e^{-}$and high frame rate of $1.5 \mathrm{kHz}$. The SAPHIRA detector is an infrared electron avalanche photodiode (e-APD) array developed by Leonardo. This detector has been shown to exhibit sub-electron read noise at high gain and can be operated at the high frame rates we require. As a proof-of-concept during the proposal stage the SAPHIRA was deployed on the SCExAO bench as the Pyramid WFS camera. Using an internal calibration source and DM generated turbulence the loop was successfully closed without modulation at a frame rate of $120 \mathrm{~Hz}$ and effective wind speed of $5 \mathrm{~ms}^{-1}$.

The SAPHIRA detector for KPIC is used in conjunction with the Pizzabox controller, developed at the University of Hawaii. The camera is used in the following configuration:

- Read-reset mode. The SAPHIRA can be readout in different modes and the read-reset mode is used as a compromise between low noise and high frame rate. ${ }^{14}$

- A frame size of $128 \times 128$ pixels. This is determined by the choice of sampling (see section 3.1 ) and optical design (see section 3.2). Along with the readout mode this sets a maximum frame rate of $1.5 \mathrm{kHz}$.

- Triggered readout. For a modulated Pyramid WFS it is important to synchronize the frame rate of the camera with the modulator speed: the integration period should be an integer multiple of the modulation period. This is achieved using a micro-controller to provide the a pulse signal to trigger the detector readout and the sine and cosine signals for the modulator.

- Avalanche gain. The avalanche gain will be set to achieve an effective read noise of at most $1 \mathrm{e}^{-}$.

\subsection{Control}

The control of the Pyramid WFS is based on the Real Time Controller (RTC) developed for SCExAO. ${ }^{15}$ This controller has been extensively developed with a Pyramid WFS AO system, and includes a library of functions for calibration and closed loop operation, allowing for easy testing of new algorithms. The Pyramid RTC is integrated with the current Keck AO RTC, which is used to control the existing tip-tilt and deformable mirrors. ${ }^{16}$

\subsection{Expected performance}

A simulation campaign of the expected performance is a key component of the design process. Figure 6 shows an example of one such simulation for the Keck Pyramid WFS. Here the expected Strehl ratio is considered, at different wavelengths over a range of magnitudes of the WFS guide star. Further simulations and details are given in Plantet et al. (2018). ${ }^{17}$

Results such as those shown in figure 6 involve optimizations of the system, including the frame rate; loop gain; modulation amplitude; and the number of modes used. This is done for each case (each guide star magnitude). In particular such simulations have suggested that the system is optimized across a range of magnitudes for modulation amplitudes of $1-2 \frac{\lambda}{D}$.

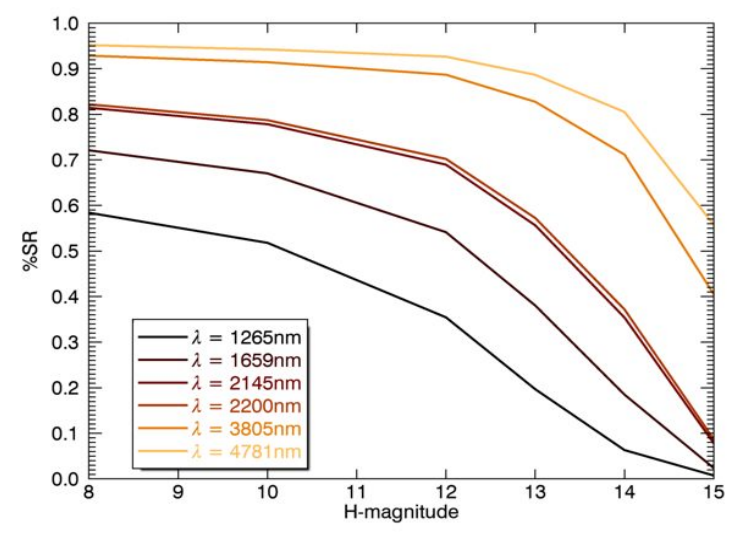

Figure 6: Expected performance (Strehl ratio) of the Keck Pyramid WFS with a readout noise of $1 \mathrm{e}^{-}$. The wavefront sensing is done in $\mathrm{H}$-band and the correction utilizes a $20 \times 20$ DM (current Keck DM). 


\section{INTEGRATION AND TESTING}

\subsection{Construction and alignment}

The KPIC system is currently installed in the laboratory at the Institute for Astronomy (IfA) in Hilo, Hawaii. The Pyramid WFS was built and aligned at the IfA, whilst the Fiber Injection Unit (FIU) was constructed at Caltech in Pasadena, California. In April 2018 the FIU was shipped to Hawaii and the two plates were integrated and co-aligned to produce the KPIC instrument. A photograph of the current system is shown in figure 7 (a). Also shown are images of the final focal planes on each system (b), with each plate demonstrating good internal alignment.

Further details on the alignment procedure can be found in Lilley et al. (2018). ${ }^{12}$ In addition to building and aligning the system various tests of the opto-mechanics have been carried out, with particular focus on calibration of the modulator and the stage for the collimating lens. All tests have verified performance within our requirements. Repeatability tests of the kinematics, including the FIU, are documented in Delorme et al. $(2018) \cdot{ }^{18}$

\subsection{First Pyramid signals}

The first Pyramid WFS signals from the KPIC instrument are shown in figure 8. The image of the four pupils (a) corresponds to the Pyramid measurement with the system aligned with the internal source mounted on

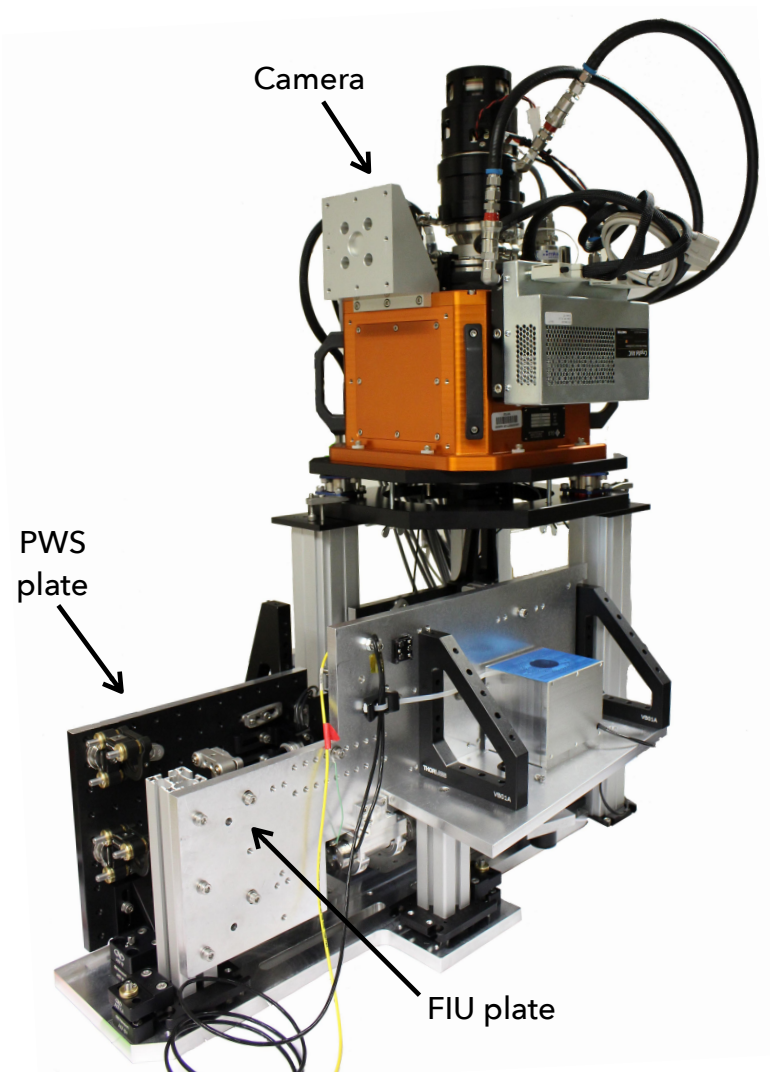

(a) KPIC laboratory setup
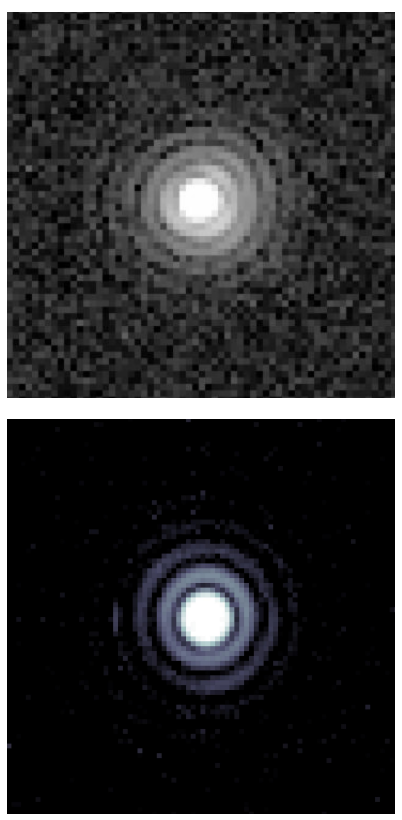

(b) KPIC focal planes at $\lambda=1550 \mathrm{~nm}$. Top: PSF on FIU tracking camera. Bottom: PSF at Pyramid WFS.

Figure 7: Current KPIC laboratory status. In operation the two plates are mounted parallel to one another on the baseplate. a: Photo of laboratory setup. The silver plate is the Fiber Injection Unit (FIU) plate and the black plate is the Pyramid WFS (PWS) plate. The SAPHIRA camera is positioned above. b: Measurements of the PSFs at the final focal plane of the two plates after co-alignment. 


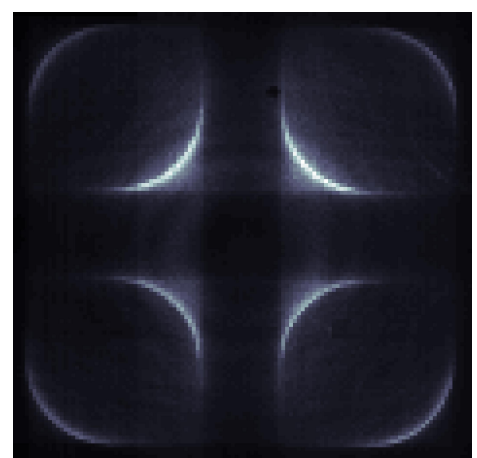

(a) Flat Pyramid WFS measurement

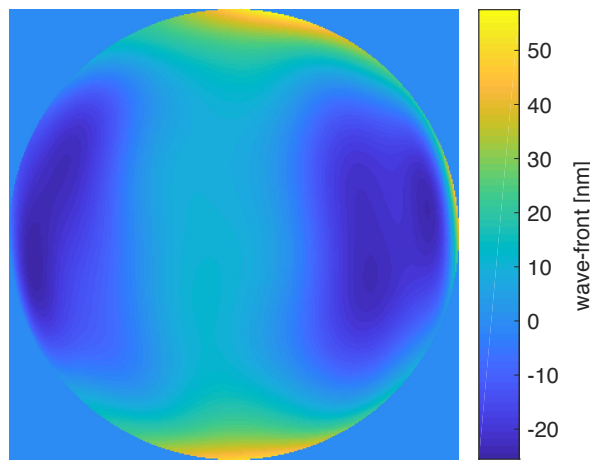

(b) Reconstructed wavefront

Figure 8: First Keck Pyramid WFS measurements. a: Flat Pyramid measurement with white light source. b: Reconstructed wavefront corresponding to $\sim 16 \mathrm{~nm}$ rms wavefront error (with tip and tilt removed).

the FIU plate. The input is a white light source, with an H-band filter before the camera restricting the wavelengths incident on the detector. The pupil images display few aberrations, confirming our confidence in the alignment. The pupil size and seperation are consistent with the design. A wavefront was reconstructed from this measurement using a simulated interaction matrix. This is also shown in figure 8 (b). The wavefront is mostly astigmatism, but exhibits are very small wavefront error of $\sim 16 \mathrm{~nm}$ rms, well within the alignment requirements.

\subsection{SAPHIRA performance}

A key test of the system is confirming the performance of the SAPHIRA detector meets the requirements of KPIC $\left(<1 \mathrm{e}^{-}\right)$. Initial measurements of the detector noise suggest effective sub-electron read noise can be achieved at a avalanche gains of $\sim 50$ in the Keck $\mathrm{AO}$ environment. Measurements of the avalanche gain for different bias voltages are shown in figure 9 . A gain of 50 is indicated by the red dashed line, illustrating that such a gain is well within the range of our detector. The gain could be pushed even further, however at very high gains $(>>100)$ some additional noise and saturation of pixels is observed.

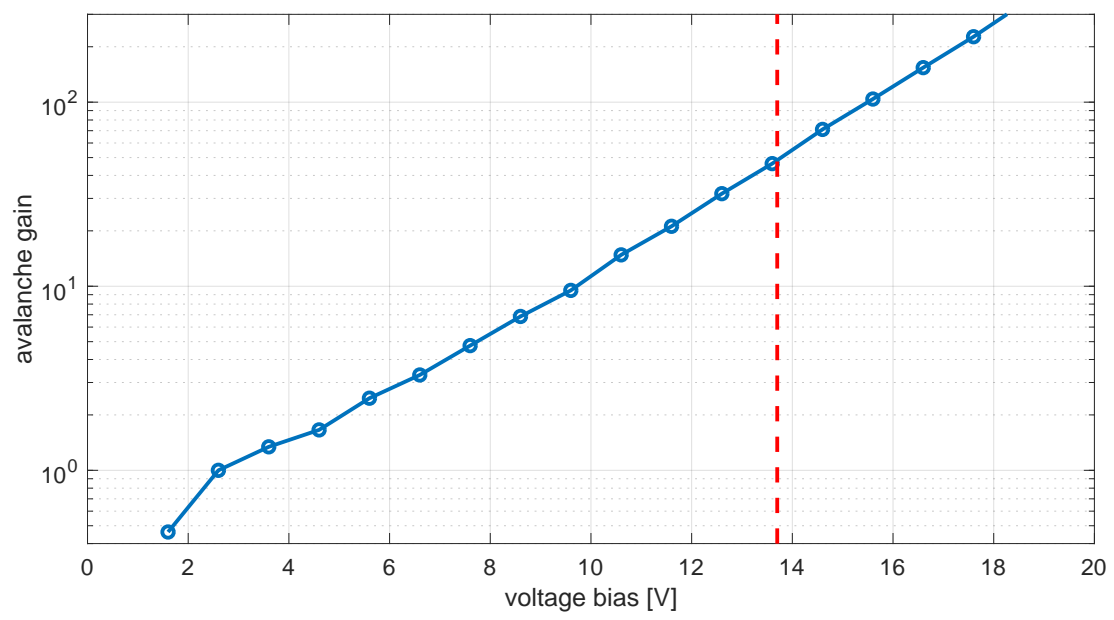

Figure 9: Measurement of avalanche gain for the SAPHIRA detector used in the Keck Pyramid WFS. The gain is measured for different voltage biases, with a gain of 50 marked by the red dashed line. 


\section{CONCLUSION}

Wavefront sensing in the infrared is desirable for particular science cases where the target is faint in the visible but bright in the infrared, such as M-type stars. The ability to use the science target as the AO natural guide star can provide the high contrasts and resolutions required to detect potential exoplanets. When considering the highly sensitive Pyramid WFS, sensing in the infrared has additional advantages over the visible, reducing non-linear effects and increasing the range of the sensor. This results in better performance at low modulation for typical atmospheric seeing and a reduced impact of non-common path aberrations.

As part of the Keck Planet Imager and Characterizer a near-infrared Pyramid WFS will be implemented on the Keck II telescope. In this paper the key design features are summarized. The system is currently assembled at the Institute for Astronomy in Hilo, Hawaii, co-aligned with KPIC's Fiber Injection Unit. The instrument has demonstrated good internal alignment, with wavefront aberrations of $16 \mathrm{~nm} \mathrm{rms}$. The crucial technology for the WFS is the SAPHIRA e-APD array, a low noise infrared detector. Initial measurements have shown the requirement of sub-electron read noise is achieved with an avalanche gain of $\sim 50$, well within the capabilities of the camera.

\section{ACKNOWLEDGMENTS}

The near-infrared pyramid wavefront sensor is supported by the National Science Foundation under Grant No. AST-1611623. The camera used with the pyramid wavefront sensor is provided by Don Hall with support by the National Science Foundation under Grant No. AST-1106391. The fiber injection unit is supported by the Heising-Simons Foundation.

The W. M. Keck Observatory is operated as a scientific partnership among the California Institute of Technology, the University of California, and the National Aeronautics and Space Administration. The Observatory was made possible by the generous financial support of the W. M. Keck Foundation.

The authors wish to recognize and acknowledge the very significant cultural role and reverence that the summit of Maunakea has always had within the indigenous Hawaiian community. We are most fortunate to have the opportunity to conduct observations from this mountain.

\section{REFERENCES}

[1] Wizinowich, P., Chun, M., Mawet, D., Agapito, G., Dekany, R., Esposito, S., Fusco, T., Guyon, O., Hall, D., Plantet, C., and Rigaut, F., "Near-infrared wavefront sensing," in [Proceedings of the SPIE], 9909 (2016).

[2] Ragazzoni, R., "Pupil plane wavefront sensing with an oscillating prism," J. of Modern Optics 43, 289-293 (1996).

[3] Vérinaud, C., "On the nature of the measurements provided by a pyramid wave-front sensor," Optics Communications 233, 27-38 (2004).

[4] Esposito, S., Riccardi, A., Quirós-Pacheco, F., Pinna, E., Puglisi, A. T., Xompero, M., Briguglio, R., Busoni, L., Fini, L., Stefanini, P., Brusa, G., Tozzi, A., Ranfagni, P., Pieralli, F., Guerra, J. C., Arcidiacono, C., and Salinari, P., "Laboratory characterization and performance of the high-order adaptive optics system for the large binocular telescope," Applied Optics 49 (2010).

[5] Esposito, S., Riccardi, A., Pinna, E., Puglisi, A. T., Quirós-Pacheco, F., Arcidiacono, C., Xompero, M., Briguglio, R., Busoni, L., Fini, L., Argomedo, J., Gherardi, A., Agapito, G., Brusa, G., Miller, D. L., Ramon, J. C. G., Boutsia, K., and Stefanini, P., "Natural guide star adaptive optics systems at lbt: Flao commissioning and science operations status," in [Proceedings of the SPIE], 8447 (2012).

[6] Jovanovic, N. et al., "The subaru coronagraphic extreme adaptive optics system: Enabling high-contrast imaging on solar-system scales," Publications of the Astronomical Society of the Pacific 127(955) (2015).

[7] Morzinski, K., Close, L., Males, J., Kopon, D., Hinz, P., Esposito, S., Riccardi, A., Puglisi, A., Pinna, E., Briguglio, R., Xompero, M., Quirós-Pacheco, F., Bailey, V., Follette, K., Rodigas, T., Wu, Y., Arcidiacono, C., Argomedo, J., Busoni, L., Hare, T., Uomoto, A., and Weinberger, A., "Magao: Status and on-sky performance of the magellan adaptive optics system," in [Proceedings of the SPIE], 9148 (2014). 
[8] Mawet, D., Wizinowich, P., Dekany, R., Chun, M., Hall, D., Cetre, S., Guyon, O., Wallace, J. K., Bowler, B., Liu, M., Ruane, G., Serabyn, E., Bartos, R., Wang, J., Vasisht, G., Fitzgerald, M., Skemer, A., Ireland, M., Fucik, J., Fortney, J., Crossfield, I., Hu, R., and Benneke, B., "Keck planet imager and characterizer: concept and phased implementation," in [Proceedings of the SPIE], 9909 (2016).

[9] Peter, D., Feldt, M., Henning, T., Hippler, S., Aceituno, J., Montoya, L., Costa, J., and Dorner, B., "Pyramir: Exploring the on-sky performance of the world's first near-infrared pyramid wavefront sensor," 122 (01 2010).

[10] Bond, C. Z., Correia, C. M., Sauvage, J. F., Hadi, K. E., Abautret, Y., Neichel, B., and Fusco, T., "Optimized calibration of the adaptive optics system on the lam pyramid bench," in [Adaptive Optics for Extremely Large Telescopes 5 - Conference Proceedings], (2017).

[11] Mawet, D. et al., "Keck planet imager and characterizer (kpic): recent results and status update," in [Proceedings of the SPIE], (10703-6) (2018).

[12] Lilley, S. et al., "A near-infrared pyramid wavefront sensor for keck adaptive optics: onto-mechanical design," in [Proceedings of the SPIE], (10703-127) (2018).

[13] Atkinson, D. E., Hall, D. N. B., Baker, I. M., Goebel, S. B., Jacobson, S. M., Lockhart, C., and Warmbier, E. A., "Next-generation performance of saphira hgcdte apds," in [Proceedings of the SPIE], 9915 (2016).

[14] Goebel, S. B., Hall, D. N. B., Guyon, O., Warmbier, E., and Jacobson, S. M., "Overview of the saphira detector for adaptive optics applications," Journal of Astronomical Telescopes, Instruments, and Systems 4, $4-4-10$ (2018).

[15] Guyon, O. et al., "The compute and control for adaptive optics (cacao) real time control software," in [Proceedings of the SPIE], (10703-51) (2018).

[16] Cetre, S. et al., "A near-infrared pyramid wavefront sensor for keck adaptive optics: real-time controller," in [Proceedings of the SPIE], (10703-119) (2018).

[17] Plantet, C. et al., "Keck ii adaptive optics upgrade: simulations of the near-infrared pyramid sensor," in [Proceedings of the SPIE], (10703-115) (2018).

[18] Delorme, J.-R. et al., "A fiber injection unit for keck: final design and first results," in [Proceedings of the SPIE], (10702-77) (2018). 